\title{
Permeability alteration by salt precipitation: numerical and experimental investigation using X-Ray Radiography.
}

\author{
Olivier Lopez ${ }^{2}$, Souhail Youssef ${ }^{2}$, Audrey Estublier ${ }^{1}$, Jostein Alvestad ${ }^{2}$, Christin Weierholt Strandli ${ }^{2}$ \\ ${ }^{1}$ IFP Energies Nouvelles, Rueil-Malmaison France \\ ${ }^{2}$ Equinor, Trondheim, Norway
}

\begin{abstract}
The injection of a gas phase through a water saturated porous medium can reduce the water saturation not only by displacement mechanisms but also by evaporation mechanisms. In the presence of brine, this process can induce salt crystallization and precipitation within the porous medium with a risk of permeability alteration. In the field of gas production and storage, the occurrence of such a phenomenon can have detrimental consequence on the well productivity or injectivity. In this work, we investigated experimentally and numerically the effect of dry gas injection on salt precipitation and permeability impairment. State of the art equipment designed for high throughput coreflood experimentation was used to capture the dynamic of salt migration using X-Ray radiography. A set of experiments have been conducted on a sample of Bentheimer sandstone (10 $\mathrm{mm}$ in diameter and $20 \mathrm{~mm}$ in length) as well as a two layers composite sample with a significant permeability contrast. Experiments were conducted using Nitrogen and $\mathrm{KBr}$ brine with different boundary conditions (i.e. with and without capillary contact). Results showed that salt precipitation results from the interplay of different parameters, namely pressure gradient, brine salinity, capillary forces and vapor partial pressure. Experimental observations indicate that in the case of dry gas injection, salt systematically precipitates but permeability alteration is observed only if a capillary contact is maintained with the brine. We built a 2D flow model integrating two-phase Darcy flow, capillary forces, salt effect on vapor partial pressure, dissolved salt transport, as well as the different PVT equilibria needed to describe properly the systems. Once calibrated, the model showed good predictability of lab scale experiment and thus can be used for parametrical study and upscaled to the well bore scale.
\end{abstract}

\section{Introduction}

In the field of natural gas production as well as gas storage in aquifer, salt deposition is considered as a potential risk for gas wells. In Northern Germany, field evidences of mainly halite precipitation have been reported during gas production [1]. A synthetic review of gas well productivity impairment by salt plugging can be found in [2]. Injection of a gas phase through a water saturated porous medium can reduce the water saturation not only by displacement mechanisms but also by evaporation mechanisms. To understand the phenomena of drying induced by gas production, Mahadevan et al. [3] did an extensive experimental and theoretical study to show that injection of vapor saturated gas (i.e., humid gas) in a rock sample can induce drying [3-4]. The origin of the drying is that the water mole fraction in the gas phase at equilibrium depends on pressure. In the context of gas storage in aquifer, experiments have been conducted by [5-6] where dry gas was injected in brine saturated sandstone samples with different salt concentrations in the brine. They showed that the drying rate is proportional to the gas velocity and that any dissolved salt in the water can be transported by capillary flows induced by drying and can even accumulate near the injection surface. The permeability can then be reduced by pore clogging. They conclude that all these mechanisms must be included in models to calculate the near wellbore permeability and porosity changes with time, to correctly determine well injectivity.

A first attempt to model the drying process in a porous media at the laboratory scale was proposed by Mahadevan et al. [4]. The authors considered two different models, one to model the first stage of the immiscible displacement, and another during the evaporation regime. The first part is described by a standard two-phase Darcy model. The second accounts for compressibility-driven drying and capillarity effects. The two models were not coupled. The evaporation model was then extended in [7] to consider the impact of capillary-driven liquid film flow on the evaporation rates as well as the rate of transport of salt through those films. TOUGH 2 family of reservoir simulators was also used by [8-9] to simulate two-phase flow of a gas mixture in equilibrium with a sodium chloride brine, including the modeling of halite precipitation/dissolution. The simulations were conducted for near well bore model in the context of $\mathrm{CO}_{2}$ injection at laboratory scale.

In this study, we present a comprehensive experimental investigation of the effect of gas flow in a sandstone sample initially saturated with water or brine. A set of experiments have been conducted on a Bentheimer sandstone sample $(10 \mathrm{~mm}$ in diameter and 20 $\mathrm{mm}$ in length) as well as on a two layers composite 
sample of different permeability (high and low) with the aim of investigating the effect of salt precipitation on permeability impairment at different boundary conditions. Experiments were then simulated using an in-house advanced compositional model (CooresFlow).

\section{Materials and methods}

\subsection{Experimental set-up}

The experimental set-up used in this study is shown in Fig. 1. Nitrogen was injected at a constant pressure using a downstream pressure regulator. Its flow rate was monitored by a Coriolis mass flow meter Brine solution was injected using an Isco Pump. The different lines were connected to an X-ray transparent hassler type cell equipped from each side with three connections (inlet, outlet and static line for the pressure transducer). A data acquisition system was used to monitor the pressure, mass flow and temperature. Finally, the set-up was installed in dedicated radiography facility equipment for in-situ saturation monitoring.
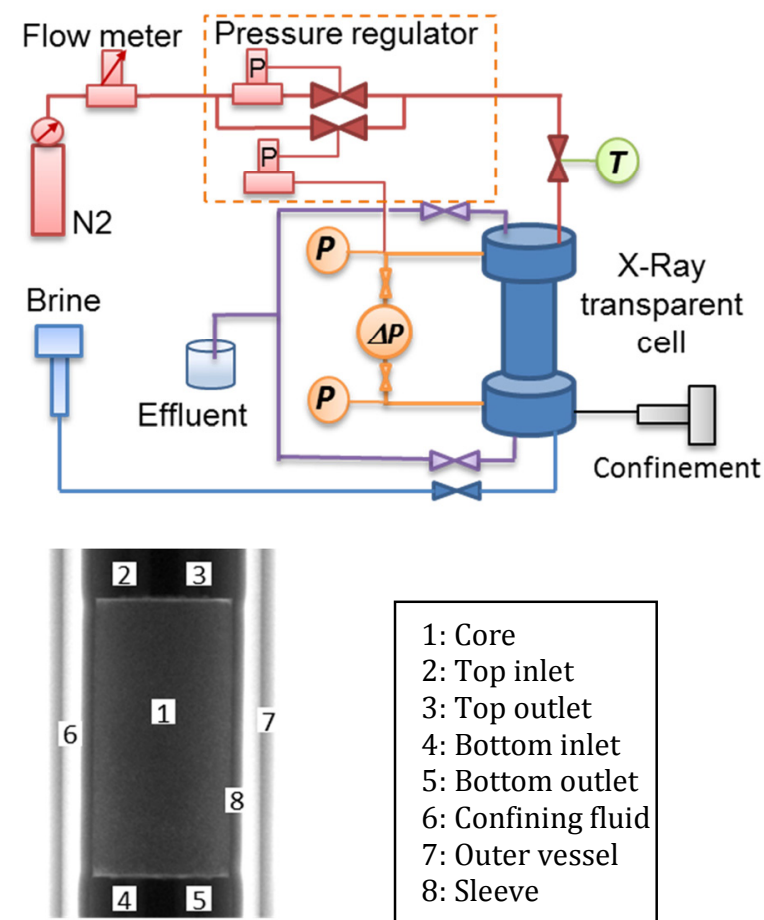

Fig. 1 Experimental set-up and radiograph showing the sample shape and the basic components of the Hassler injection cell.

During fluid injection, the sample was imaged using an X-ray radiograph equipment. The system consists of an X-ray source, a flat panel and a data processing unit for computation, visualization and data analysis. The radiographs were captured with a resolution a $20 \mu \mathrm{m}$ at a rate of one frame per second. Radiographs were then cropped to show only the sample (see zone 1 in Figure 1). The stack of images was processed to monitor water saturation and salt concentration evolution during gas injection [10]. Indeed, image gray levels are proportional to the transmitted X-ray intensity which is defined by the
Beer Lambert's law in the case of a multicomponent material as:

$$
I_{t}=I_{o} e^{-\left(\mu_{b t} X_{b}+\mu_{g} X_{g}\right)+\alpha}
$$

where $I_{o}$ and $I_{t}$ are respectively the incident and the transmitted X-ray intensity, $\mu_{b t}$ and $\mu_{g}$ are respectively the brine and the gas attenuation coefficients, $X_{w}$ and $X_{g}$ are respectively the brine and the gas portions in the linear path of the X-ray and $\alpha$ is the attenuation of the structure. Considering two references states, $100 \%$ brine saturated and dry images the above equation can be written as:

$$
I_{b}=I_{o} e^{-\mu_{b 0} X+\alpha} \text { and } I_{g}=I_{o} e^{-\mu_{g} X+\alpha}
$$

where $\mathrm{X}$ is the linear path of the X-ray that corresponds to the total porosity and $\mu_{\mathrm{b} 0}$ is the initial brine attenuation coefficient.

By construction we obtain:

$$
X_{b}+X_{g}=X
$$

Afterward, normalizing the X-ray intensity $\mathrm{I}_{\mathrm{t}}$ by both the $100 \%$ brine and the dry case, and considering $\mu_{b} \gg \mu_{g}$ we obtain:

$$
\begin{aligned}
& \qquad \frac{\ln \left(\frac{I_{b}}{I_{0}}\right)-\ln \left(\frac{I_{t}}{I_{0}}\right)}{\ln \left(\frac{I_{b}}{I_{0}}\right)-\ln \left(\frac{I_{g}}{I_{0}}\right)}=\frac{-\mu_{b 0} X+\mu_{b t} X_{b}}{-\mu_{b 0} X} \\
& \text { When salt concentration is constant we have: }
\end{aligned}
$$

$$
\mu_{b t}=\mu_{b 0} \text { and } \frac{X-X_{b}}{X}=S_{g}
$$

where $S_{g}$ is the gas saturation. If the salt concentration changes, $\mu_{b t}$ will vary with an upper limit equal to the $\mathrm{KBr}$ attenuation coefficients. If we normalize the $\mathrm{X}$-ray transmitted intensities (i.e. relative X-ray absorption) by the mean intensity of a dry sample with precipitated salt, we can assess qualitatively the variation of salt quantity compared to the initial distribution.

\subsection{Experimental procedure}

A set of experiments has been conducted using the set-up described above. The experiments have been conducted on the Bentheimer sandstone sample and the Bentheimer/Molière composite core. Four steps have been applied to investigate the effect of salt on the drying dynamics and the effect of capillary contact on salt accumulation and permeability alteration. the sequence of injection consisted in:

1-Saturating the sample with the wetting phase by injecting the water (or brine) from the bottom inlet and opening the top outlet (the bottom outlet and top inlet are closed). To ensure a full saturation, we have imposed a back pressure of 4 bar and monitored the saturation by $\mathrm{X}$-ray.

2-Lowering the pore pressure to 1 bar, opening the bottom outlet and top inlet and closing the top outlet. The water injection is maintained allowing a sweeping of the bottom face of the sample.

3-The gas is then injected at a constant pressure. The variation of the saturation and the local concentration of salt is monitored by the X-ray radiographs and the gas flow rate is monitored by the Coriolis flow meter. The 
injection is stopped once no more variations are observed on the radiograph and the flow rate.

4-The sample is finally cleaned by injecting deionized water until the reference X-ray absorption is reached.

\subsection{Sample properties and fluids}

The experiments were conducted using two consolidated water-wet sandstones: Bentheimer, and Molière. Bentheimer sandstone is a high porosity (23\%) permeable $(1350 \mathrm{mD})$ rock composed mainly of quartz; while Molière sandstone is a medium porosity (14\%) with very low permeability $(0.05 \mathrm{mD})$ composed of quartz and pore filled clay.

In the three first experiments a Bentheimer core of 1 $\mathrm{cm}$ in diameter and $2 \mathrm{~cm}$ in length was used. In the last experiment and to reproduce a lateral capillary contact parallel to the flow direction, a composite sample was shaped using Bentheimer and Molière sample. The plugs were first half cut in the longitudinal direction using a diamond saw. The two half pieces were then gathered by using a nonwoven fabric reinforced by a wet ceramic powder (see Fig. 2 a) to improve capillary contact. The Bentheimer half core is made shorter than the Molière one to avoid outlet capillary contact with the distributor (see Fig. 2.b)

(a)
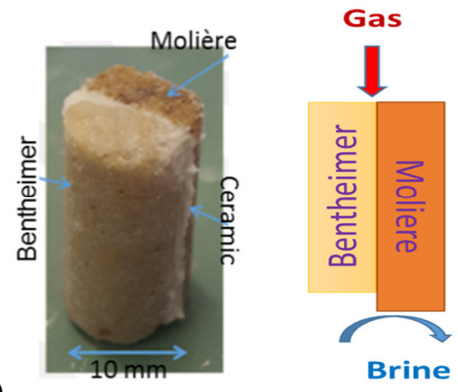

Fig. 2 (a) Photographs of the composite core composed of Bentheimer and Molière half cores contacted with nonwoven fabric reinforced by a wet ceramic powder. (b) Sketch of the composite sample during fluids injection.

To perform the different experiments, we have used dry nitrogen and $100 \mathrm{~g} / \mathrm{KBr}$ brine. $\mathrm{KBr}$ salt was chosen as it has a high electronic density allowing a good X-ray contrast with water and gas. The solubility limit of $\mathrm{KBr}$ in water at $20^{\circ} \mathrm{C}$ is $560 \mathrm{~g} / 1$.

\section{Experimental observations}

As pointed out in the introduction, drying and salt precipitation result from the interplay of different parameters. To separate the effect of these parameters, we have conducted a non-exhaustive number of experiments that focus on the effect of salt on permeably alteration with three different boundary conditions: no capillary contact at the outlet face, capillary contact at the outlet by brine seeping and lateral capillary contact using a composite sample as described in the previous section. In the following, we discuss the impact of these parameters.

\subsection{Effect of salt}

To study the effect of salt on permeability alteration, we have conducted two experiments where we have first injected dry gas in a Bentheimer sample initially saturated with deionized water (Exp 1). The purpose of this first experiment is to observe the drying dynamics without the interference of the salt. The inlet relative gas pressure was set to 100 mbar. The pressure drop was chosen to be above the entry pressure of $70 \mathrm{mbar}$ and corresponds to a pressure gradient of $5 \mathrm{bar} / \mathrm{m}$ which is representative of near well bore pressure gradient. Fig. 3 shows the evolution of the mean gas saturation within the sample as well as the mass flow rate of the gas for Exp 1.

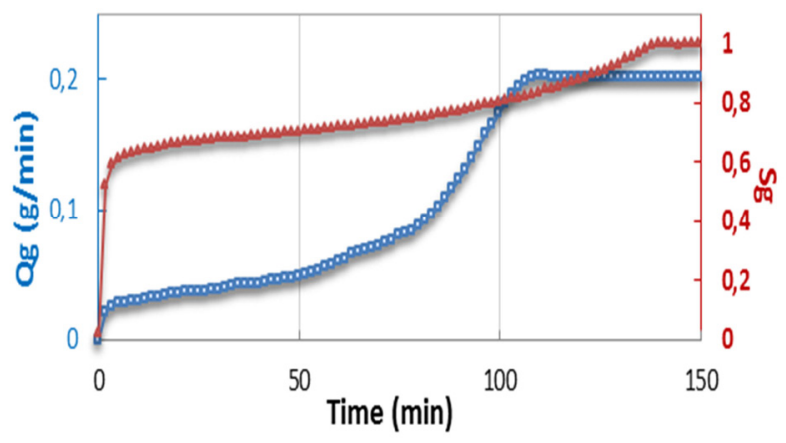

Fig. 3 Exp 1 Gas saturation (red curve) and mass flow rate (blue curve) evolution as a function of time for gas injection in sample initially saturated with deionized water.

Four regimes can be distinguished:

- $\quad$ In the first few minutes (between 0 and $10 \mathrm{~min}$ ) the gas drains the sample until it reaches the residual water saturation that was established to $40 \%$. Fig. 4 shows the local saturation of the sample after $10 \mathrm{~min}$. The longitudinal profile as well as the $2 \mathrm{D}$ saturation map show a typical drainage profile with a quite constant saturation on the first $2 / 3$ of the sample (around $30 \%$ of water) followed by an increasing water saturation toward the outlet due to capillary end effects.

The next regime (between 10 and $70 \mathrm{~min}$ ) exhibits a smooth increase in the gas saturation that reaches $74 \%$ as well as in the flow rate that establishes at $35 \%$ of the final rate (cf. Fig. 3). The profile at $70 \mathrm{~min}$ displays a constant shift of the initial profile. This observation can be explained by an apparent homogeneous drying all along the sample which means that the equilibrium between the gas and the water is not instantaneous. Or by an equilibrium reached between the water evaporation and capillary forces that tends to redistribute liquid from regions of high-water saturation to region of low-water saturation.

Following this second regime that ends at 70 min and at water mean saturation of $26 \%$ we observe a noticeable increase in the gas flow rate until $110 \mathrm{~min}$ as well as in the gas saturation until $140 \mathrm{~min}$. This change in the drying rate corresponds to the progression of a discontinuity and a sharp front on the saturation profile and 2D map. This front propagates through the sample 
toward the outlet allowing a subsequent increase in the flow rate until it reaches a plateau.

The final stage corresponds to the dry state (above $140 \mathrm{~min}$ ). It is to be noticed that the flow rate reaches its limit before the gas saturation.
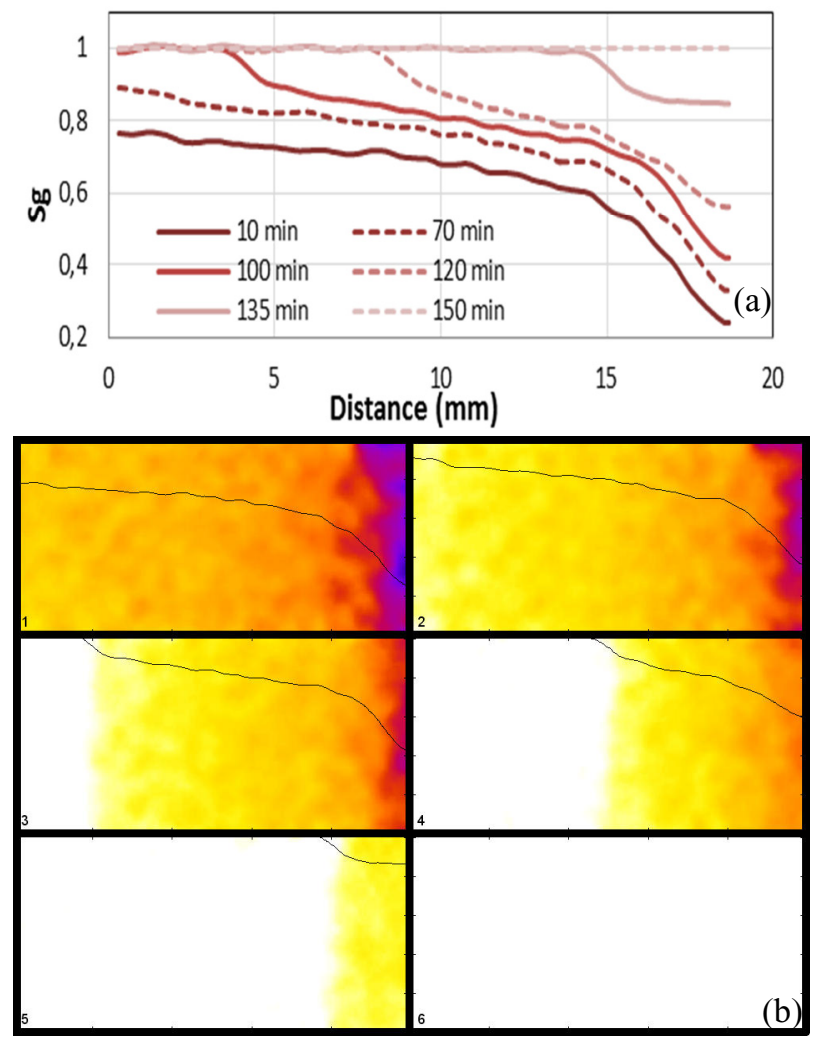

Fig. 4 Exp 1 evolution of gas saturation (a) 1D profile et (b) 2D map as a function of time (gas is injected from left) in sample initially saturated with deionized water.

This first experiment shows that injecting a dry gas in a porous media containing only deionized water allows to evaporate the total content of water without altering the permeability of the sample. The observation of the saturation profiles shows that the drying effect takes place first at the inlet of the sample and then propagates throw a sharp front downstream. The two first regimes involved in this particular case are most likely an instantaneous phase equilibrium between gas and water coupled with a capillary equilibrium generated by the gas flow and the water saturation.

The drying scenario would probably be first the gas vaporizes water at the entrance of the sample creating a capillary disequilibrium and decreasing the water saturation. This results in water redistribution by counter-current imbibition to compensate for the water evaporation at the inlet. Once the mean water saturation reaches a minimum (26\%), the capillary disequilibrium cannot be compensated anymore. The evaporation front appears and propagates in the sample until the total drying of the sample.

In a second experiment (Exp 2) dry gas was injected in the same sample initially saturated with $\mathrm{KBr}$ brine at a relative gas pressure of 150 mbar. Fig. 5 shows the evolution of the gas flow rate as a function of time as well as the relative X-ray absorption that was normalized by the final stage of Exp 3. The flow rate exhibits the same behavior as for the first experiment (i.e. a first increase corresponding to the drainage phase, a smooth increase slope followed by a more pronounced rise till flow rate reaches a constant plateau corresponding to the dry state). The relative X-ray absorption that corresponds in this particulate case to the mean gas saturation shows an asymptotic behavior with a smooth increase of the gas saturation. The final flow rate did not show any evidence of permeability alteration due to salt precipitation.

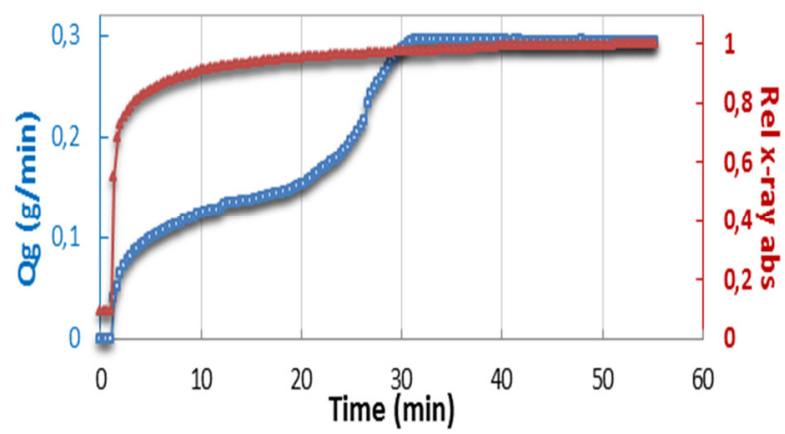

Fig. 5 Exp 2 X-ray relative absorption (red curve) and gas mass flow rate (blue curve) evolution as a function of time for gas injection in sample initially saturated with $100 \mathrm{~g} / 1 \mathrm{KBr}$ brine.

The observation of the local profiles shows a more complex behavior (see Fig. 6). As the relative X-ray absorption was normalized by the gray level of the final sate, the profile can be qualitatively interpreted as the relative variation in salt localization (i.e. when the profile goes over or under one, it means that the quantity of salt decreases or increases respectively). Thus, we can observe that the salt concentration slightly decreases in the first half of the sample while it considerably increases at a specific location situated $5 \mathrm{~mm}$ from the outlet, corresponding to the location of the capillary end effects.

This behavior can be explained by the competition between drying and capillary rise. Actually, as drying decreases the saturation from the inlet toward the outlet as shown by case 1, capillary forces tend to equilibrate the saturation by displacing brine toward the inlet. As a consequence, the salt concentration locally increases and once it reaches the solubility limit, it precipitates.

The measured gas flow rate shows that, even if salt has precipitated, it did not affect the permeability. This could be explained by the low quantity of salt initially present in the sample after the drainage. An estimation of the initial volume of dissolved salt after the drainage gives a value $0.003 \mathrm{cc}$ which corresponds to $0.01 \mathrm{PV}$ (PV is the pore volume). This reduction of the pore volume does not seem sufficient to reduce the absolute permeability. To confirm this observation, a 3D images of the sample was taken at initial dry state and after salt precipitation. Fig. 7 shows a slice picked at the level of maximum salt precipitation. We can distinguish almost no difference between the two images apart few bright points corresponding to solid salt in some locations. 

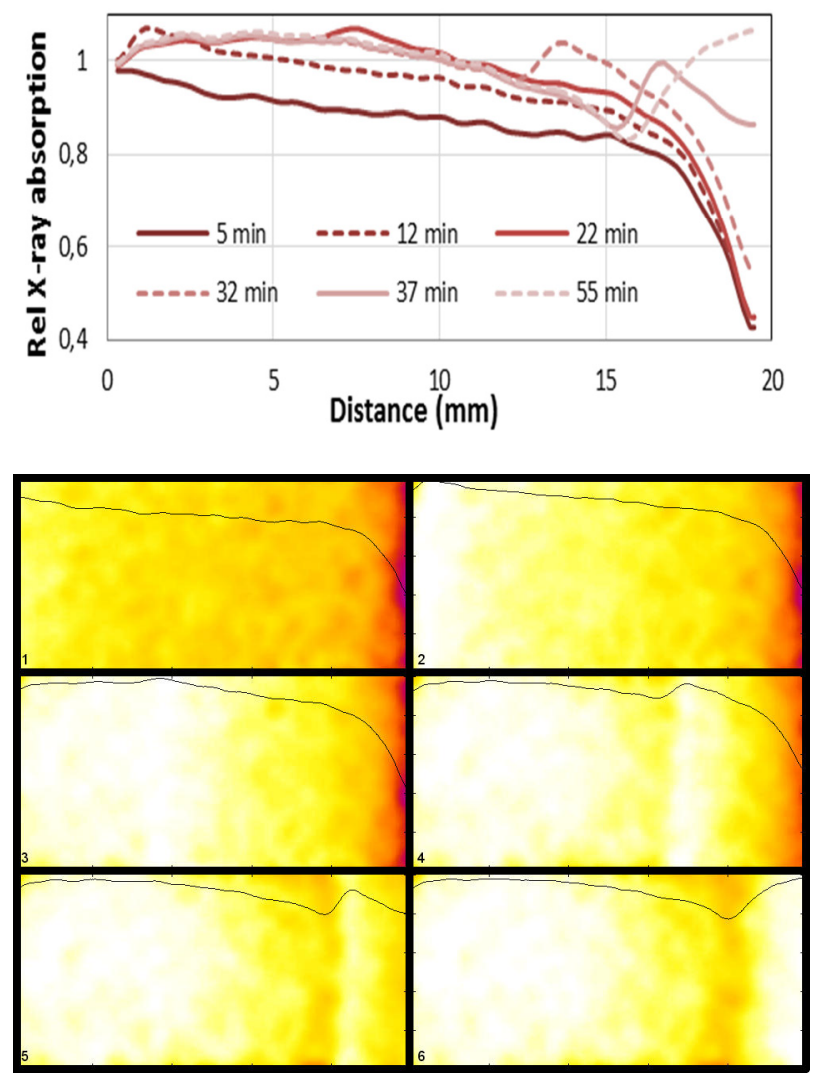

Fig. 6 Exp 2 evolution of X-ray relative absorption (a) 1D profile and (b) 2D map as a function of time (gas is injected from left) in sample initially saturated with $100 \mathrm{~g} / 1 \mathrm{KBr}$ brine.

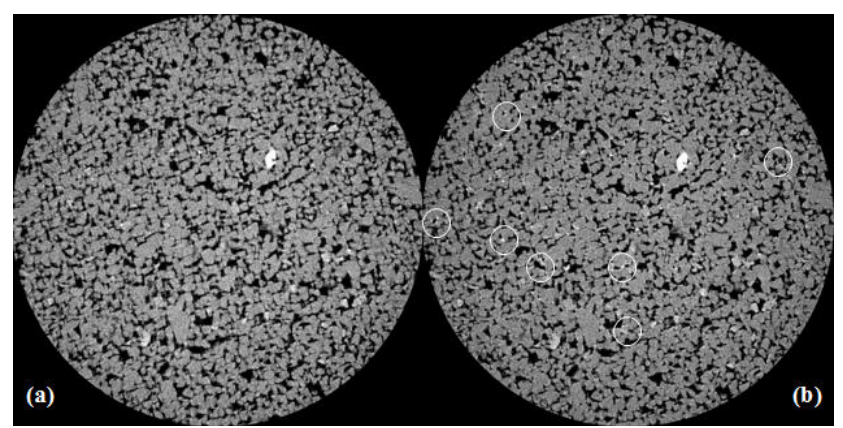

Fig. 7 2D slices picked at the maximum salt precipitation location ( $4 \mathrm{~mm}$ from the outlet) (a) at the initial dry state and (b) at the final state of the experiment corresponding to a dry state with precipitate salt. Some locations where salt has precipitated are indicated by circles.

\subsection{Outlet capillary contact effect}

To investigate the capillary effect, dry gas was injected in a sample initially saturated with a $100 \mathrm{~g} / \mathrm{l} \mathrm{KBr}$ brine while contact with brine was maintained by sweeping the bottom face of the sample at a relative gas pressure of 150 mbar. Fig.8 shows the evolution of the gas flow rate and the relative X-ray absorption as a function of time. In this case, we observe a completely different behavior than in the previous cases. After an initial increase due to drainage, the gas flow rate experiences successive decline and rise cycles with increasing amplitude before it reaches zero after six hours of injection. The relative X-ray absorption shows a constant decrease which corresponds to an increase of the sample density. This observation suggests an accumulation of additional salt within the sample that is supplied by the brine in contact with the bottom face of the sample.

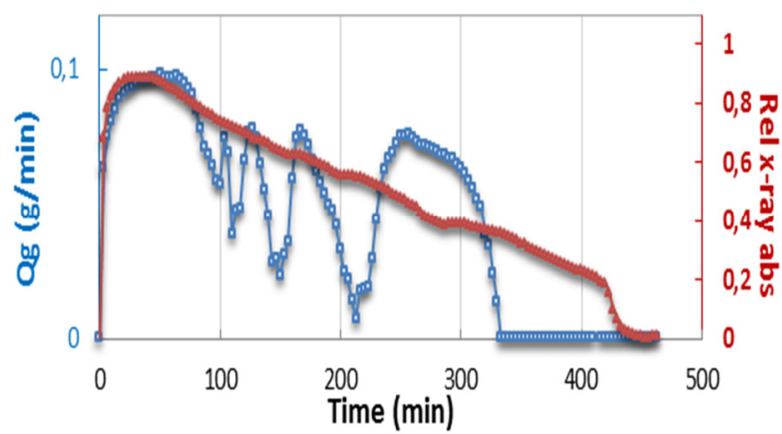

Fig. 8 Exp 3 X-ray relative absorption (red curve) and gas mass flow rate (blue curve) evolution as a function of time for gas injection in sample initially saturated with $100 \mathrm{~g} / \mathrm{KBr}$ brine and with outlet capillary contact.

The profiles illustrated in Fig. 9 give more insight into the mechanisms that take place during this experiment. We observe first a local decrease in the relative X-ray attenuation at $8 \mathrm{~mm}$ from the inlet at 63 min that suggests an increase in salt concentration. The advent of this salt strip is correlated with a decrease in the gas flow rate and corresponds most probably to salt precipitation that alters permeability to gas. We observe then an alternation of dilutions and precipitation corresponding respectively to the increase and the decline of the gas flow rate with an intensification of the relative X-ray attenuation along the salt strip. Finally, we observe a displacement of the salt strip during the successive cycles of dilution precipitation toward the inlet of the sample. At the end of the experiment the salt strip is located $5 \mathrm{~mm}$ far from the inlet.

The observed phenomena can be explained by the competition between drying and capillary rise. Indeed, the gas flow dries out the sample, which is manifested by a decrease in the water saturation. However, as we have an active capillary contact, capillary suction compensates for the decrease of water saturation to maintain a capillary equilibrium, which in turn leads to an increase of local salt concentration. At one point the salt concentration is sufficiently high to precipitate salt and altering the gas permeability. As the gas flow rate declines, the capillary rise becomes predominant, allowing the dilution of the precipitated salt and a regeneration of the gas permeability. The alternation of this instable equilibrium leads to an increase of the precipitated salt after each stage till a point of no return is reached where the volume of precipitated salt is sufficiently high to stop the gas flow and the capillary suction.

At the end of the experiment a 3D image of the sample was taken to evidence salt precipitation. Fig. 10 shows a slice picked up at the maximum salt precipitation location. The accumulation of solid salt is clearly observable all over the porous space of the sample (white part). The solid salt plugs the pores and is responsible of the permeability alteration. 

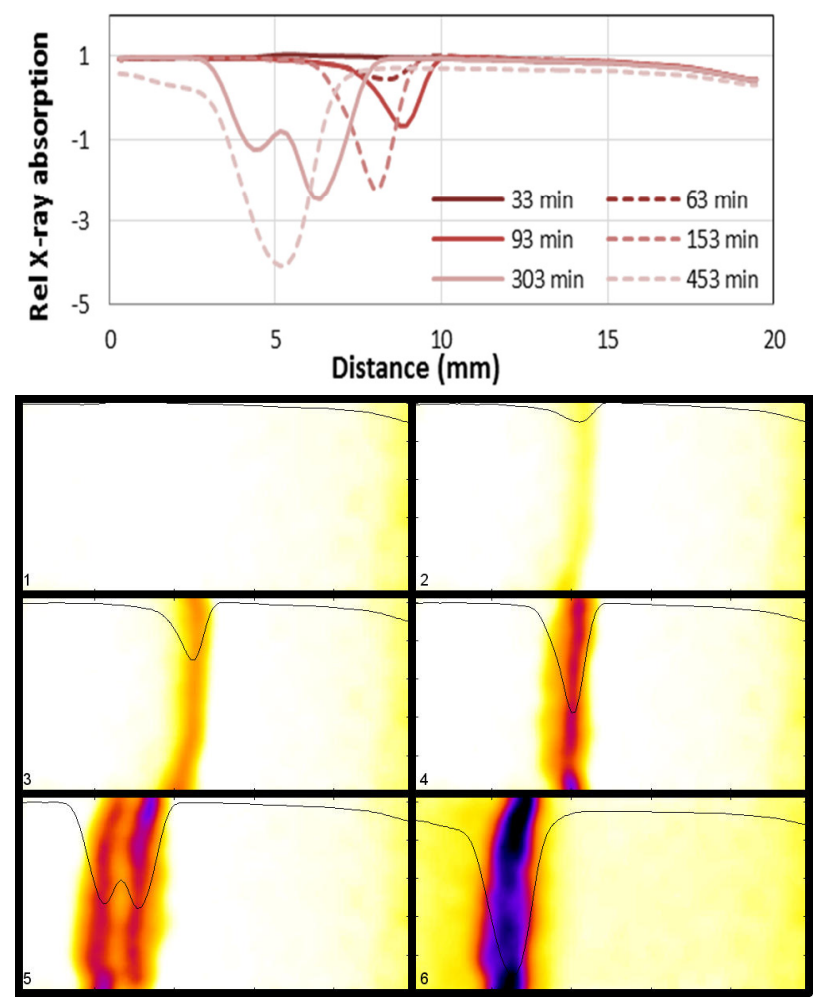

Fig. 9 Exp 3 X-ray relative absorption (a) 1D profile and (b) 2D maps (gas is injected from left) as a function of time for dry gas injection in sample initially saturated with $100 \mathrm{~g} / \mathrm{KBr}$ brine and with outlet capillary contact.

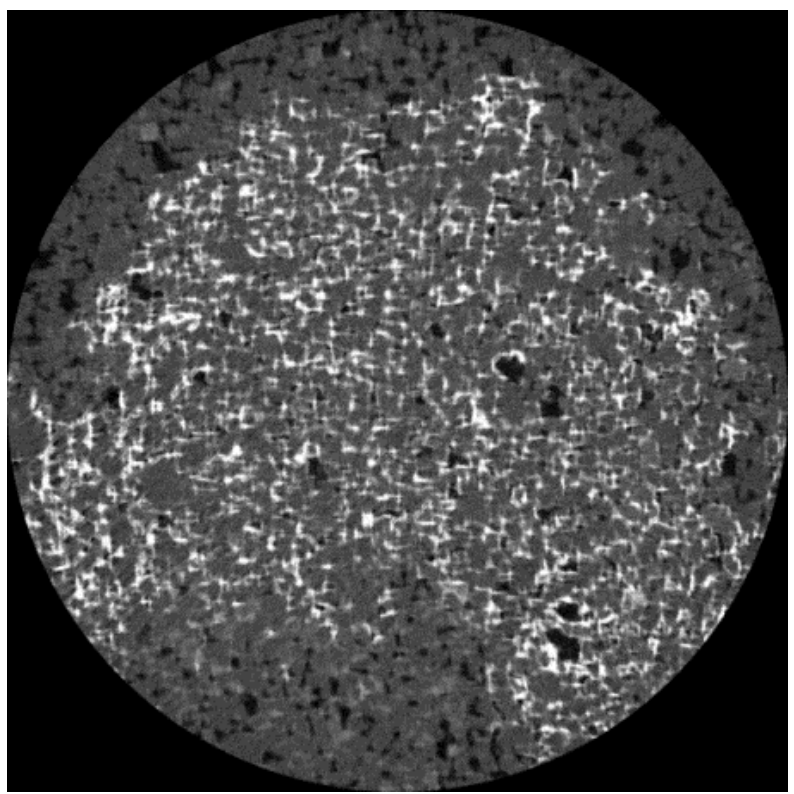

Fig. $102 \mathrm{D}$ slice picked at the maximum salt precipitation location (5 $\mathrm{mm}$ from the inlet) at the final state of the experiment. Light gray level corresponds to solid salt that has precipitate due to gas drying out.

\subsection{Lateral capillary contact effect}

In this last experiment (Exp 4), we have injected dry nitrogen in the composite sample initially saturated with $100 \mathrm{~g} / \mathrm{KBr}$ brine. The inlet gas pressure was set to 450 mbar (the outlet was opened to atmosphere). Fig. 11 shows the evolution of the gas flow rate as a function of time as well as the relative X-ray absorption that was normalized by the finale stage within the Bentheimer half core. We can observe that when gas is injected, the relative X-ray absorption and the gas flowrate rise rapidly to a maximum that correspond probably to the residual water saturation state. Following this first stage, we note a monotonous decrease in the relative X-ray adsorption which means salt accumulation within the sample. As consequence, the gas flow rate decreases which corresponds to a progressive permeability alteration.

Fig. 12 shows the $1 \mathrm{D}$ and 2D locale relative X-ray absorption. The Bentheimer half core is positioned on the upper part of the images. The first image at $26 \mathrm{~min}$ shows that when gas is injected, a preferential path is created throw the Bentheimer sample that goes to Swi whereas the Molière half core remains fully brine saturated. The second image shows the advent of salt precipitation that is located almost at the middle of the sample and near the Bentheimer and sleeve boundary. However, the shape of the precipitate salt zone is completely different from the one observed in the previous experiment. Indeed, we do not observe a strip perpendicular to the gas flow direction but rather an arrow like spot. The next images show that salt precipitates and accumulates in parallel to the flow direction. As the Bentheimer half plug is only in contact with the Moliere one, this observation confirms that the lateral capillary contact is active and can provide enough salt to plug completely the permeable zone.

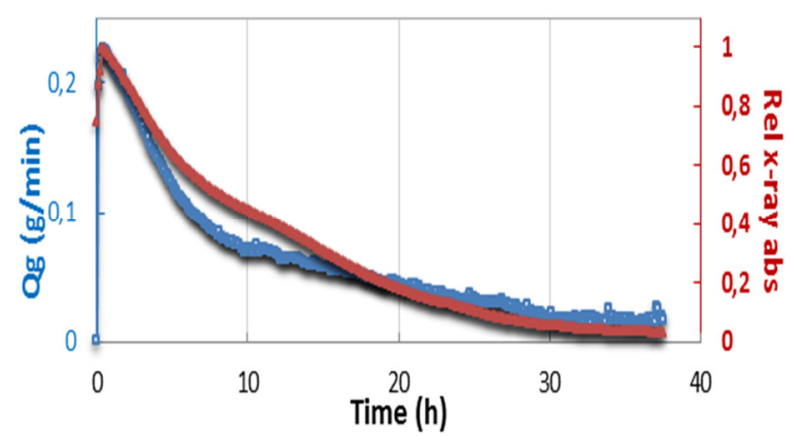

Fig. 11 Exp 4 X-ray relative absorption (red curve) and gas mass flow rate (blue curve) evolution as a function of time for gas injection in sample initially saturated with $100 \mathrm{~g} / \mathrm{KBr}$ brine and with outlet capillary contact.

To better observe the shape of the salt precipitate zone, a 3D image was taken at the end of the experiment. Fig. 13 shows 2D sagittal and axial images of the sample. We can clearly see the solid salt barrier that cause permeability alteration. It is interesting to note that this barrier lies in diagonal to the Bentheimer sample and extend on the two third of the length. This shape reveals the complexes interplay of the different mechanisms involved in salt precipitation.

In this case, the drying front is opposed not only to parallel capillary forces coming from the capillary end effect in the Bentheimer half core (as described in Exp 2) but also to perpendicular capillary forces resulting from 
the contact with the Moliere half core. This configuration can then explain the shape of the precipitated salt that results from the competition between the two perpendicular drying fronts.
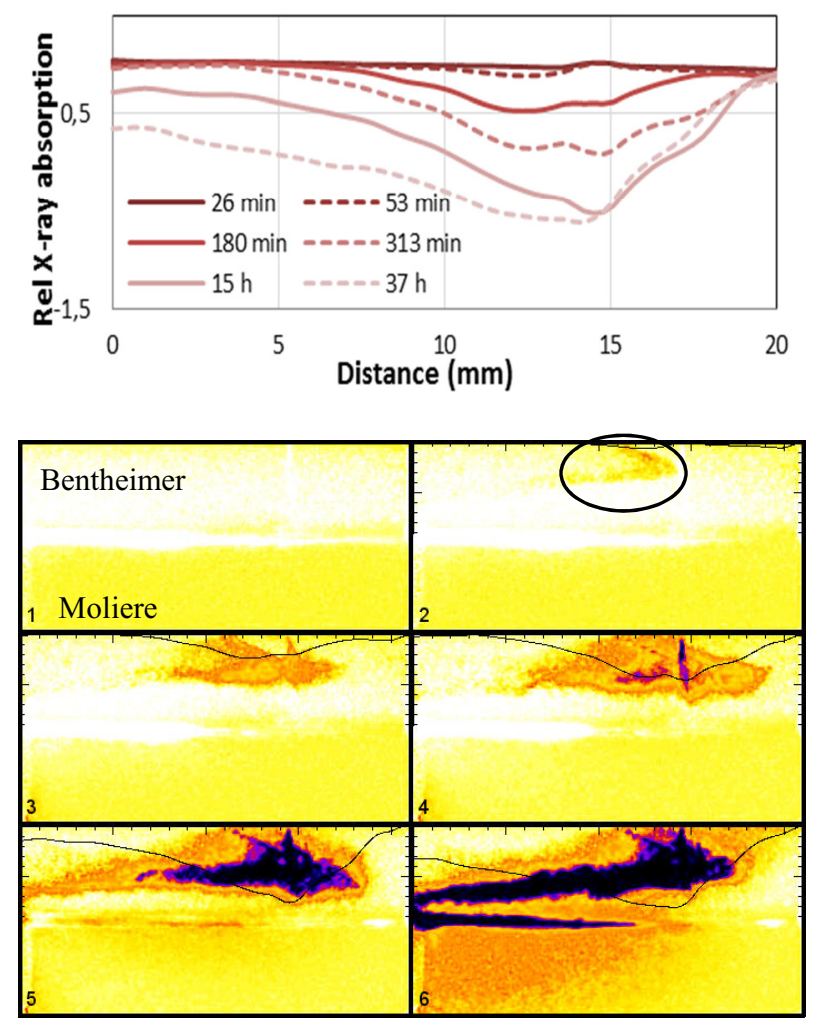

Fig. 12 Exp 4 X-ray relative absorption (a) 1D profile and (b) 2D maps (gas is injected from left) as a function of time for dry gas injection in sample initially saturated with $100 \mathrm{~g} / \mathrm{l} \mathrm{KBr}$ brine and with lateral capillary contact.

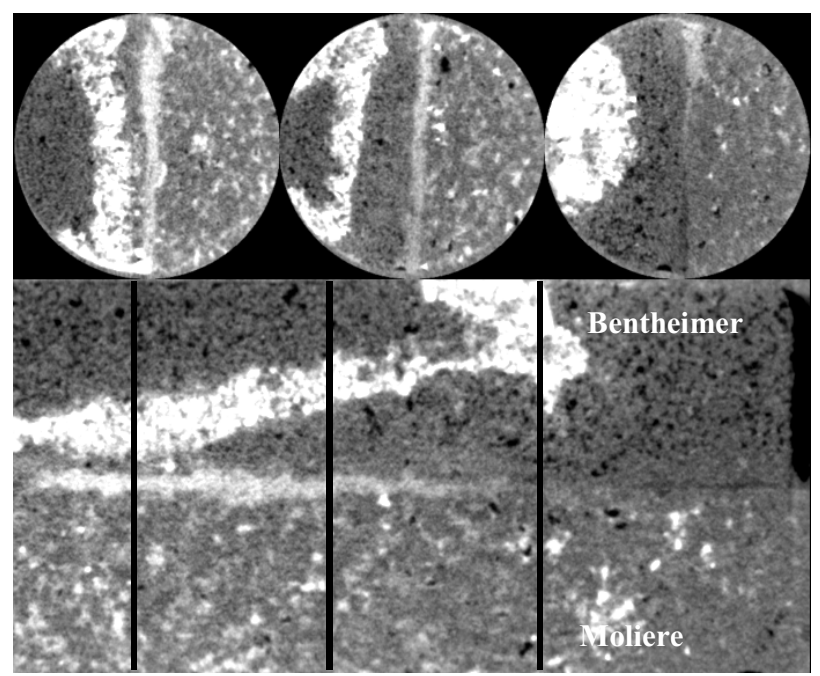

Fig. 13 2D sagittal slice (bottom) and 2D axial slices picked at three different salt precipitation location at the final state of the experiment. Bright zone corresponds to solid salt that has precipitated due to gas drying out.

\section{Numerical interpretation}

In this study we used CooresFlow an in-house simulator designed for reactive flows in porous media. It was developed to model multiphase transport processes coupled with fluid-rock interactions, for various applications including $\mathrm{CO}_{2}$ underground storage as well as enhanced recovery of hydrocarbon reservoirs.

\subsection{General characteristics}

CooresFlow is a THC (thermal hydro-chemistry) simulator that consider the following phenomena:

- multiphase flow in porous media, with viscous and capillary forces,

- transport of chemical components by advection, diffusion and dispersion,

- transfers within the fluids or on the surface of the rock governed by local equilibrium,

- chemical equilibrium or kinetic reactions to describe geochemical exchanges,

- dynamic modification of the porosity and permeability of the porous medium over time.

In the case of gas production or storage, it allows to consider:

- the phenomena of mass transfer between liquid water and water vapor (drying),

- the transport and precipitation of dissolved species (salts),

- the coupling between porosity and permeability changes.

The mathematical formulation used in the simulator is a generalization of the compositional formulation based on material and energy balance equations and chemical equilibrium equation (for more see [11])

\subsection{Physical and numerical model}

In this study we consider a multiphase compositional system composed of four phases: two fluids (water and gas) and two solids (rock and salt). As the system is compositional, each phase can contain one or several species. In the studied case, the species are:

- $\mathrm{H} 2 \mathrm{Ow}$ and $\mathrm{KBrw}$ (the dissolved salt) in water,

- $\mathrm{N} 2 \mathrm{~g}$ (nitrogen) and $\mathrm{H} 2 \mathrm{O}$ vapor $(\mathrm{H} 2 \mathrm{Og}$ ) in gas,

- Precipitated salt (KBrs) in salt.

- The Rock phase (Quartz) considered inert in this study (no exchange with other phases)

The rock sample is modelled by a vertical parallelepiped of 100 cells in $\mathrm{Z}$ direction. Its sizes are $0.86 \mathrm{~cm}$ in $\mathrm{X}$ and $\mathrm{Y}$ directions and $1.98 \mathrm{~cm}$ vertically. Initially, the sample is saturated with either deionized water or a $100 \mathrm{~g} / 1 \mathrm{KBr}$ brine. The temperature is constant within the sample and does not evolves with time. The pressure is also initially constant and equal to the atmospheric pressure. The boundary conditions were defined in accordance to the experimental design.

The thermodynamic model computes the density and viscosity of the fluids. The brine density evolves with the salt concentration ( $\mathrm{KBr}$ molar fraction) using a linear 
relation based on experimental data from the Detherm 2015 database, for a temperature of $25^{\circ} \mathrm{C}$. In the same way, the brine viscosity evolves with the salt concentration by interpolating linearly experimental data of the Detherm 2015 database for a temperature of $25^{\circ} \mathrm{C}$. The gas properties are assumed constant since the pressure is closed to 1 bar and the humidity rates are low $(<2 \%)$. We consider a gas density of $1.2 \mathrm{~kg} / \mathrm{m} 3$ and viscosity of $1.75810^{-5}$ Pa.s.

In this work, two phase transfers are modelled: Water evaporation and condensation, and salt dissolution and precipitation. The salt dissolution/precipitation is considered instantaneous. It is modelled by an equilibrium constant with a linear dependency on temperature. The equation is based on the experimental data of the Detherm 2015. The water evaporation/condensation is also considered instantaneous. This hypothesis is confirmed by a Peclet number higher than 1 that means the water advection dominates the water diffusion in gas. The model is defined by a table of equilibrium constants with a dependency to temperature, pressure and salt concentration. This table is based on experimental data from the Carnot and Detherm 2015 databases.

\subsection{Flow through drying modelling}

To generate the petrophysical model, we have first considered a homogeneous sample with a constant porosity of $23 \%$ and permeability to water of $1450 \mathrm{mD}$. Relative permeabilities and capillary pressure were then estimated by history matching the mean saturation profiles and gas flow rate end point from Exp1. The resulting relative permeability to water and to gas as well as water/gas capillary pressure are illustrated in Fig. 14. It is to be noted that the resulting absolute permeability to gas was estimated to $2450 \mathrm{mD}$ which is much higher than the water permeability. This can be explained by the inertial effect. This effect was not considered in this study. Irreducible water saturation was estimated to $12 \%$. In the domain of 0 to $12 \%$ of water saturation, the relative permeability to gas is found to be constant while the capillary pressure increases slightly.

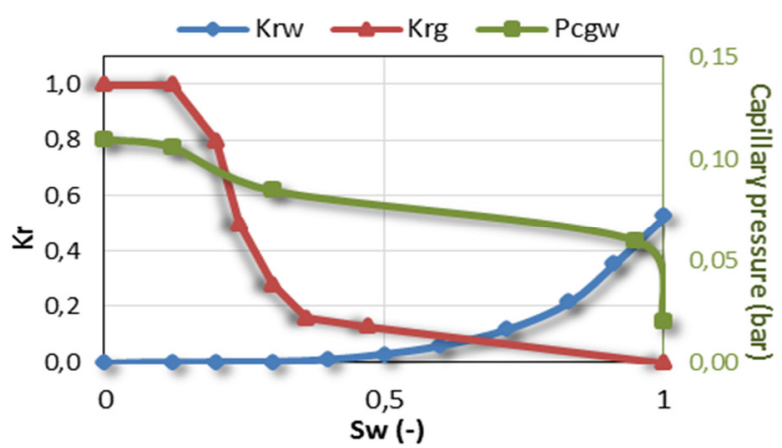

Fig. 14 Water (Krw), gas (Krg) relative permeability and Gaswater capillary pressure (Pcgw) versus water saturation

By comparing the simulation results with the experimental data in the case of Exp 1 (see Fig. 15), we observe that the simulated system exhibits the same behavior than the experimental case. Indeed, we observe the same behavior of the gas rate with an initial important increase corresponding to the drainage phase, then a smoother increase followed by a greater increase during the drying phase within the sample until the gas rate reaches a plateau

The model succeeds to fit properly the mean saturation and gas flow rate end point as well as the major part of saturation profiles. However, differences are observed on the gas flow rate dynamic. This discrepancy can be explained by the uncertainties on capillary effects and saturation at the outlet boundary that induce a difference on the gas saturation profiles near the outlet. Nevertheless, the reactive transport model succeeds to simulate the same physical phenomena such as the drying effect, the capillary counter-current, the disappearance of a phase with similar characteristic times.

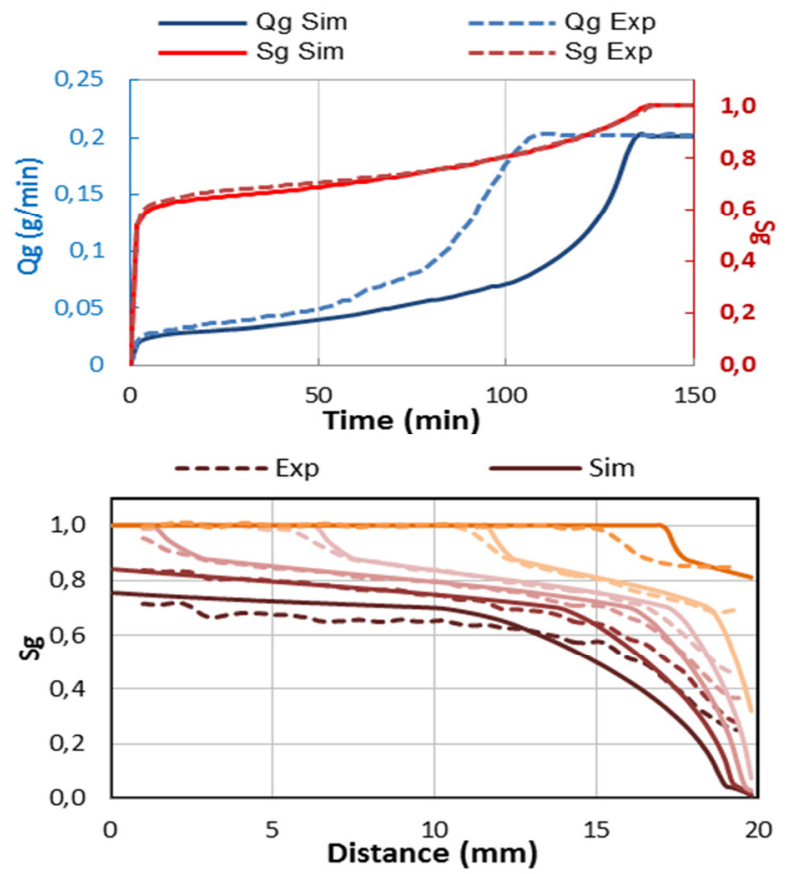

Fig. 15 Comparison of the experimental and simulated results of Exp 1 (dry gas injection in sample initially saturated with deionized water)

\subsection{Salt precipitation modelling}

To consider the effect of salt precipitation on permeability to gas, the porosity is allowed to evolve over time with the volume of precipitated or dissolved salt. The permeability can change accordingly using a Kozeny Carman model with critical porosity:

$$
k=k_{0}\left(\frac{\emptyset-\emptyset_{c}}{\emptyset_{0}-\emptyset_{c}}\right)^{a}\left(\frac{1-\emptyset_{0}+\emptyset_{c}}{1-\emptyset+\emptyset_{c}}\right)^{b}
$$

Where $k$ and $\varnothing$ are respectively dynamic permeability and porosity, $k_{0}$ and $\emptyset_{0}$ are respectively initial permeability and porosity and $\emptyset_{c}$ is a critical porosity beyond which permeability is null. Exponents $a$ and $b$ as well as $\emptyset_{c}$ were history matched using data from Exp 3. 
Best match was found with 0.08 for $\emptyset_{c}, 2.5$ for a and 1.5 for $b$. Experiment 2 was simulated using the same model parameters defined in experiment 1 apart from the initial saturation where the salt concentration was set to $100 \mathrm{~g} / \mathrm{l}$ and the relative gas pressure at the inlet of 150 mbar.

Fig. 16 shows the evolution of the simulated (continuous line) gas flow rate, mean gas saturation within the sample and the inverse of the normalized $\mathrm{KBr}$ quantities. Experimental data (dashed line) were added for comparison. Simulated gas flow rate shows the same behaviour as for previous case with also a slower dynamic than the experimental data due probably to the same reason mentioned previously. However, comparison of simulated gas saturation and relative $\mathrm{X}$ Ray attenuation is not straightforward as X-Ray attenuation is sensitive not only to water saturation but also to salt quantities. Still the two quantities decrease monotonically which agrees with the increase of the XRay attenuation. We also observe that the salt quantity reaches a plateau after about $20 \mathrm{~min}$ indicating that no more salt is evacuated from the sample which mean that no more water convection is active. This time corresponds also to a change in the flow rate slop indicating a change in the water transport mechanism which becomes dominated by the evaporation and drying mechanisms.

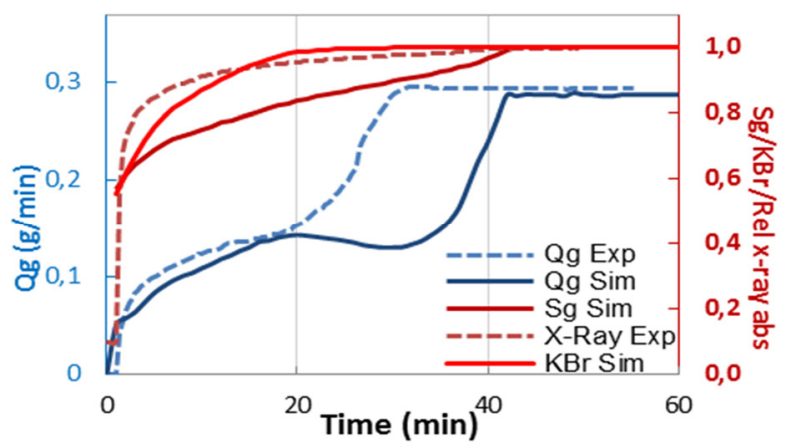

Fig. 16 Comparison of the experimental and simulated results of Exp 2 (dry gas injection in sample initially saturated with $100 \mathrm{~g} / 1$ brine)

Simulation results show also a similar $\mathrm{KBr}$ salt profile evolution with the experimental data as well as a salt accumulation near the outlet of the sample. Fig. 17 shows four different profiles taken at four different time and corresponding to (a) experimental X-Ray attenuation normalized by the final mean X-Ray attenuation, (b) the $\mathrm{KBr}$ quantity along the sample normalized by the final one, (c) dissolved $\mathrm{KBr}$ concentration and (d) gas saturation along the sample. For each date, we observe on the $\mathrm{KBr}$ quantity a front similar to the experimental data that separates the precipitated-salt zone from the dissolved-salt zone. This front has a characteristic shape that is induced by the combination of an increasing water saturation (cf. Fig. 17.d) with a decreasing $\mathrm{KBr}$ concentration (cf. Fig. 17.c) that reaches a maximum at the interface between the dry zone and the wet zone. The front moves towards the outlet at the same velocity as the evaporation front. It is to be noted that simulation shows no evidence of overconcentrated zone (i.e. zone where concentration is greater than solubility limit). At
$55 \mathrm{~min}$, the sample is completely dried and all the $\mathrm{KBr}$ salt is precipitated. Within the sample, the $\mathrm{KBr}$ amount corresponds to the amount that was included in the residual water saturation (after $20 \mathrm{~min}$ ). But near the outlet boundary, we observe an accumulation of salt. It results from the initial capillary end effect and very close to the outlet, from the competition between the drying effect and the capillary re-imbibition.

This salt precipitation occurs when the solubility threshold is reached. It reduces the porous volume in proportion with the solid $\mathrm{KBr}$ density. The final average porosity was estimated at 0.228 with a minimum of 0.225 which is very closed to the initial 0.23 value. The initial salt amount of the sample is not sufficient to drastically reduce the porosity. This porosity decrease induces a small permeability decrease that has a little effect on the gas rate. The average permeability to gas was $2000 \mathrm{mD}$ compared $2450 \mathrm{mD}$ at the initial state.

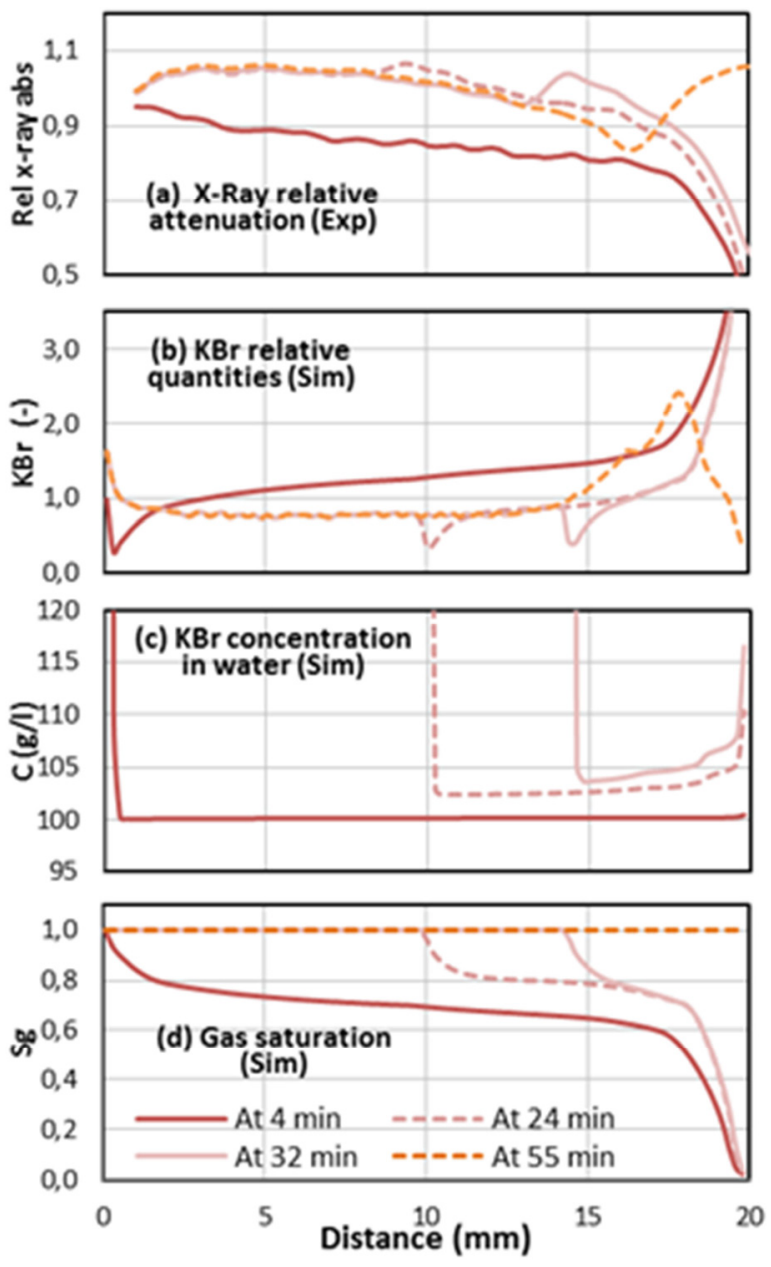

Fig. 17 Comparison of (a) experimental X-Ray attenuation profiles normalized by the final mean X-Ray attenuation and simulated profiles of (b) the $\mathrm{KBr}$ quantity along the sample normalized by the finale one, (c) the dissolved $\mathrm{KBr}$ concentration and (d) the gas saturation along the sample at four different date for Exp 2 . 


\subsection{Capillary contact modelling}

To simulate capillary contact effect, the numerical model was modified by adding an infinite water reservoir in contact with the outlet boundary to reproduce Exp 3 boundary condition. Water was set free to flow through the outlet in the two directions. In the two cases dry gas is injected in a sample initially saturated with a $100 \mathrm{~g} / 1 \mathrm{KBr}$ brine.

Fig. 18 (a) shows that the simulated gas flow rate for the third experiment (Exp 3) was comparable to the experimental one. Indeed, we observe four successive cycles of gas flow rate increase and decrease corresponding drainage/dissolution (increasing flow rate) and drying/precipitation phase (decreasing flow rate). The gas rate decreases for the last time at $327 \mathrm{~min}$ and reaches a value close to zero.

To get more insight in the cycling behavior, we have reported in Fig. 18 (b) the simulated mean gas saturation within the sample, the precipitated solid $\mathrm{KBr}$ quantities normalized by the maximum $\mathrm{KBr}$ quantity and the $\mathrm{KBr}$ concentration in water normalized by the initial concentration. Experimental data corresponding to the X-Ray normalized attenuation was added for comparison. The $\mathrm{KBr}$ curve shows clearly that the quantity of precipitate salt is increasing over time during each cycle with alternation of stagnation intervals that correspond to gas rate local minima. During those stagnation periods, we observe also a decrease in gas saturation as well as a slight increase in the brine concentration that is probably due to solid salt dissolution. However, as we do not observe any significant decrease in the solid $\mathrm{KBr}$ quantity curve, this means that during the stagnation period, an equilibrium is reached between dissolution and precipitation reaction. Salt is dissolved at the contact of brine and precipitate at the drying front.

The simulated $\mathrm{KBr}$ normalized quantity profiles at four different date are illustrated in Fig. 19. They show where salt forms a deposit. The four times correspond to the four gas local minimum rates. At these times, the salt amount is mainly located in the first $7 \mathrm{~mm}$ of the sample with a tendency to move towards the inlet over time. It is characterized by one or several peaks with an increasing base. At these zones, the porosity and the permeability are altered and reach a minimum value of respectively $9 \%$ and 1-10 mD. All these figures clearly show a correlation between the increase of the salt amount, the permeability alteration and the decrease of the gas rate.

The following process can explain the different observations made above. As in the case 2, the dry gas injection locally induces a decrease of water saturation due to water evaporation and a capillary re-imbibition from the high-water saturation zone to the low-water saturation zone to compensate the water loss. While the porous medium dries, the brine salt concentration increases until it reaches the solubility threshold. Then, it precipitates with an impact on the porosity and the permeability. The gas front on the saturation profile continues to propagate towards the outlet, leaving behind it, a dried porous medium containing a small amount of precipitated salt. At a specific location defined by the competition between the drying effect and the capillary re-imbibition, a salt accumulation starts, fed by the capillary contact, and alters the petrophysical properties. In that case, the gas rate strongly decreases until the capillary re-imbibition becomes preeminent. Then, the brine supplied by the capillary counter-current partially dissolves the precipitated salt. Indeed, the salt dissolution quickly saturates the brine that cannot dissolve an additional amount of salt. Consequently, the porosity and the permeability are also partially restored, and the gas rate increases again, until a maximum reduction. This is the end of the first cycle. Then, the process starts again with altered petrophysical conditions that impact the competition between the drying effect and the capillary re-imbibition. It explains the displacement of the salt accumulation observed in Fig. 19. The cycling process continues and stops when the pressure forces dominate the capillary forces and block the capillary flow.

In conclusion, the simulations results show a similar gas rate evolution with the experimental data as well as a salt accumulation that behaves in the same way. The reactive transport model succeeds to simulate the drying effect and the salt precipitation associated to a petrophysical properties change. The dynamic permeability model used in this study reveals adapted to model the main effects of salt precipitation.
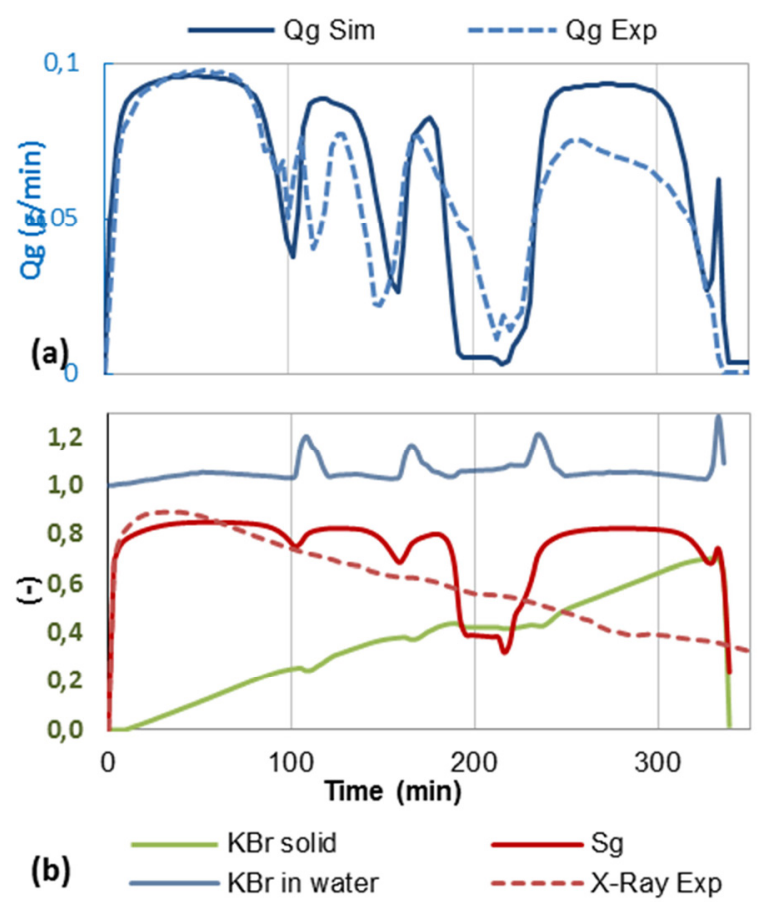

Fig. 18 Comparison of (a) experimental and simulated gas flow rate, (b) experimental X-Ray attenuation normalized by the final mean X-Ray attenuation and simulated evolution of (b) solid (precipitated) $\mathrm{KBr}$ quantity normalized by the finale total $\mathrm{KBr}$ quantity, the dissolved $\mathrm{KBr}$ concentration and the mean gas saturation Exp 3. 


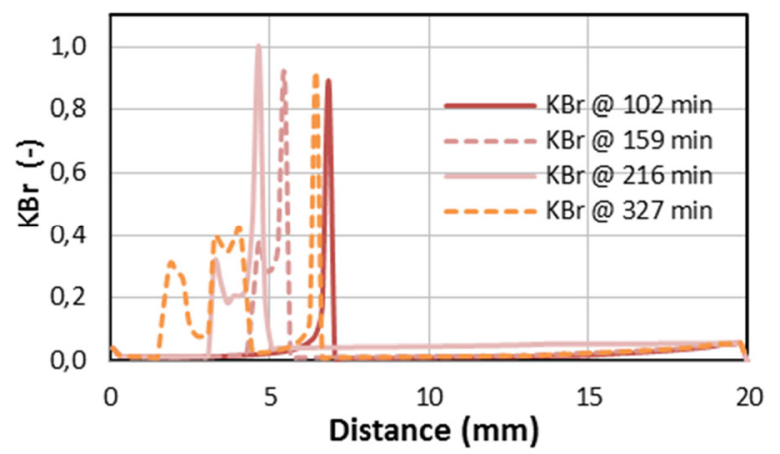

Fig. $19 \mathrm{KBr}$ quantity profile along the sample normalized by the maximum quantity at four different date for Exp 3.

In the case of Experiment 4 a two-layers mode parallel to the pressure gradient, (see Fig. 2 (b)) was built with high permeability contrast. In this case outlet capillary contact was only maintained for the low permeability layer. Fig. 20 shows the evolution of the simulated gas flow rate for Exp 4. We observe the same behavior the experimental case (see Fig. 11). The curve strongly increases due to the drainage phase then quickly decreases probably due to an alteration of the permeability. However, and as for experiment 1 and 2, the simulated flow rate exhibits a faster dynamic that can also be explained in this case by the uncertainty on the capillary contact description.

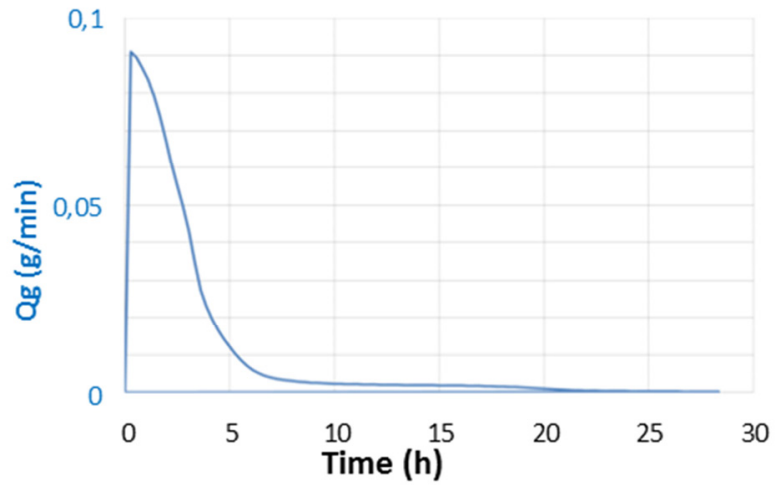

Fig. 20 Simulated gas flow rate in the case of Exp 4.

Within the property maps shown in Fig. 21, we observe the result of the longitudinal capillary contact effect. The third experiment has shown that the capillary contact at the bottom of the sample induces a salt deposit that is perpendicular to the fluid flow. In the case of Exp 4, the result is different. The re-distribution of brine from the high-water saturation zone to the low-water saturation zone mainly occurs near the contact surface between the two porous media, that induces a $2 \mathrm{D}$ profile of salt deposit that spread over a diagonal in the Bentheimer half core. As in the previous case, the brine feeding induces a salt accumulation that strongly alters the porosity (decrease from $23 \%$ to $8.06 \%$ ) and the permeability (decrease from $1350 \mathrm{mD}$ to $1.2 \mathrm{mD}$ ). The sample is plugged in 7 hours.

This simulation case shows that the plugging of a permeable rock can be caused by the combination of the drying and a brine supply coming from a low- permeability rock that contains enough volume of mobile brine.
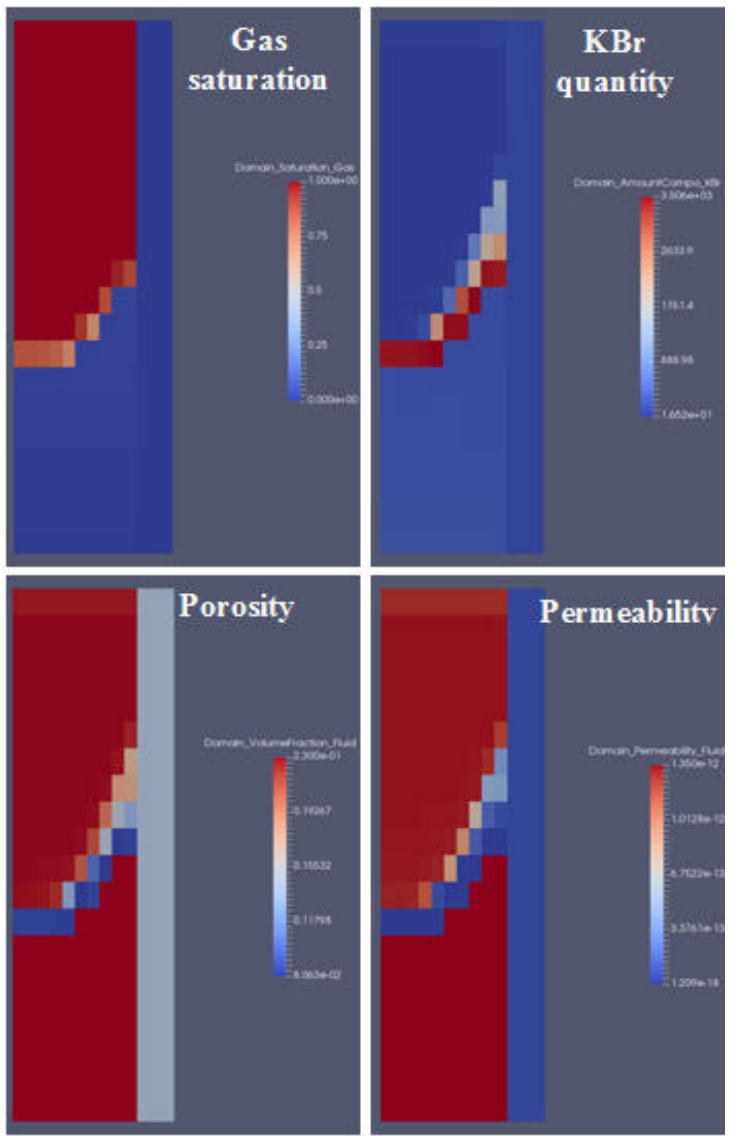

Fig. 21 2D map simulation results of gas saturation, $\mathrm{KBr}$ quantity, porosity and permeability within the sample at 25 hours for Exp 4. The low permeable zone representing the Moliere is on the left zone of the maps.

\section{CONCLUSIONS}

In this study, we have shown the importance and interplay of different parameters that govern brine drying and salt precipitation in porous media subject to dry gas injection. When dry gas is injected in a sample saturated with brine, the water is dried out near the inlet first and then a drying front propagate toward the outlet. As a consequence, salt precipitates in the porous media and can lead to pore plugging. The pore plugging will either completely block the pore space or heavily reduce the rock permeability depending on the boundary conditions.

Indeed, the experimental study showed that dry gas injection combined with a capillary water contact can trigger salt precipitation and possibly block the pore space. These experiments establish the basis for simulation models that can be used to integrate the different physical phenomena that take place during water displacement, evaporation and possibly precipitation.

Results from the experimental study have been modeled using an in-house simulator to interpret the drying and salt precipitation mechanisms. The governing equations consider various coupled mechanisms like flow-through drying, capillary forces, salt displacement, 
salt precipitation and porosity/permeability alteration. This reactive transport model succeeded in simulating the main effects observed in experimental cases:

- drying of the sample by dry gas injection, and salt precipitation

- salt accumulation supplied by capillary reimbibition until plugging of the sample,

- $\quad$ successive alteration and improvement cycles of the petrophysical properties due to precipitation and dissolution of salt.

Future work includes:

- improving the simulation approach by integrating

o modeling of hysteresis in the drainage/imbibition curves of relative permeability and capillary pressure,

- modeling of the capillary pressure evolution with porosity,

- modeling of the thermal transfer due to evaporation,

- investigating the effect of other parameters like pressure gradient, salt concentration and/or gas relative humidity to assess the induced risk of salt precipitation on permeability alteration,

- adaptation and application of the model to near well and reservoir scale problems. In particular, radial flow conditions imply rapidly changing pressure gradients close to wellbore, contrary to the uniform pressure gradient characterizing linear flow in a core.

\section{References}

1. Kleinitz W., Dietzsch G., Köhler M. (2003) Halite scale formation in gas-producing wells; Trans. IChemE, 81(A), pp352-358.

2. Aquilina, P. (2012) Impairment of Gas Well Productivity by Salt Plugging: A Review of Mechanisms, Modeling, Monitoring Methods, and Remediation Techniques, paper SPE 158480 prepared for presentation at the SPE Annual Technical Conference and Exhibition, San Antonio, Tx, 8-10 October.

3. Mahadevan J., Sharma M.M., Yortsos Y.C. (2006) Flow-Through Drying of Porous Media; AiChE Journal ; 52(7); pp2367-2380

4. Mahadevan J., Sharma M.M., Yortsos Y.C. (2007) Water removal from porous media by gas injection: experiments and simulation; Transp Porous Media; 66; pp287-309

5. Peysson Y., Bazin B., Magnier C. Kohler E., Youssef S. (2011) Permeability alteration due to salt precipitation driven by drying in the context of $\mathrm{CO} 2$ injection, Energy Proceedia (4) p4387-43941

6. Peysson Y., André L, Azaroual M. (2013) Well injectivity during $\mathrm{CO} 2$ storage operations in deep saline aquifers 1: Experimental investigation of drying effects, salt precipitation and capillary forces, Int. Journal of Greenhouse Gas Control, 22, pp 291-300
7. Le D., Hoang H., Mahadevan J. (2009) Impact of capillary-driven liquid films on salt crystallization, Transp Porous Med; 80; pp229-252

8. Giorgis T., Carpita M., Battistelli A. (2007), 2D modeling of salt precipitation during the injection of dry $\mathrm{CO} 2$ in a depleted gas reservoir, Energy Conversion and Management, 48, pp1816-1826

9. André L., Peysson Y., Azaroual M., (2014) Well injectivity during $\mathrm{CO} 2$ storage operations in deep saline aquifers - Part 2: Numerical simulations of drying, salt deposit mechanisms and role of capillary forces, Int. Journal of Greenhouse Gas Control, 22, pp301-312

10. Youssef S., Mascle M., Peysson Y., Vizika O. (2017) CAL-X: An X-Ray Radiography tool for high throughput coreflood experimentation. Applications in the EOR context. To be presented at Int. Sym. of the Society of Core Analyst Vienna, Austria

11. Gassara O., Estublier A., Garcia B., Noirez S., Cerepi A., Loisy C., Le Roux O., Petit A., Rossi L., Kennedy S., Brichart T., Chiquet P., Luu Van Lang L., André Duboin F., Gance J., Texier B., Lavielle B., Thomas B., (to be submitted), The Aquifer-CO2 Leak project: numerical modeling for the design of a $\mathrm{CO} 2$ injection experiment in the saturated zone of the SaintEmilion (France) site. 